\title{
Promoting physical activity as a healthy habit through quality physical education: Does knowledge on habitual behaviours help?
}

Francis Ries

Departamento de Education Física y Deporte, Universidad de Sevilla, España

fries@us.es

\begin{abstract}
Childhood and adolescent obesity have become one of the most important challenges to overcome in the 21st century. Practiced regularly, physical activity (PA) has significant benefits for health in youth, such as normal growth and a reduction of the risk of suffering from obesity and associated health problems. The role of habits for the engagement in PA are discussed. Understanding how habits are developed may help comprehending that PA can become habitual. Furthermore, that knowledge is useful for designing interventions to rise the levels of participation in PA for health campaigns (promoting PA maintenance for long-term health) and NCT prevention.

PE also plays an important role in the promotion of PA among children and adolescents, especially when quality physical education is implemented.
\end{abstract}

KEYWORDS

motivation; behavioural change; complex behaviours; physical education; health

DOI

10.14712/23366052.2020.7

(c) 2020 The Author. This is an open-access article distributed under the terms of the Creative Commons Attribution License (http://creativecommons.org/licenses/by/4.0), which permits unrestricted use, distribution, and reproduction in any medium, provided the original author and source are credited. 


\section{INTRODUCTION}

Youth overweight and obesity have developed as one of the most important challenges to overcome in the 21st century. It's a global problem and it is increasingly touching numerous nations, especially in metropolitan locations. The prevalence among children and adolescents aged 5-19 has augmented significantly from only 4\% in 1975 to more than $20 \%$ in 2018. 41 million of children under the age of five are overweight (WHO, 2019). Overweight and obese children and adolescents have a high probability staying obese as adults and more likely to acquire noncommunicable diseases (NCT, e.g. diabetes and cardiovascular diseases) at a younger age. Overweight and obesity, as well as the associated diseases, are mostly avoidable. Therefore, prevention of childhood and adolescent obesity needs high priority (Ng et al., 2014).

Lifestyle is characterized as the behaviour of an individual or community and it refers to routinely repeated behaviour in daily life. Lifestyle behaviours include a sequence of healthy habits: physical activity (PA), adequate diet, no tobacco and no alcohol, etc. Lifestyle is mainly influenced via the creation of a hierarchy of values and needs but also by tradition, customs and trends (Galan-López, Sánchez-Oliver, Ries, \& González-Jurado, 2019).

Practiced on a regular basis, PA has important benefits for health in children and adolescents, such as normal growing and a reduction of the risk of suffering from obesity and associated health problems (Galan-López et al., 2020). Physically active children and adolescents show higher academic achievement, better classroom conduct, more ability for concentration, and less non-attendance than their unfit peers. WHO (2010) determines that both physical and mental health benefits are attained when children and adolescents should practice at least 60 minutes of medium to vigorous intensity physical activity per day.

Schools are contemplated as one of the favourite intervention settings for promoting daily PA in children and adolescents. Physical education (PE) classes are a perfect scenario for fostering a healthy lifestyle and create PA habits (Ferriz, González-Cutre, Sicilia, \& Hagger, 2016; Solmon, 2015). In order to make PE curricula significant for young people, innovative learning theories and novel insights of PE must to be studied, assessed and applied (European Commission, 2008). Considerable current data shows a decline in the attitudes of children and adolescents towards PE because of the supremacy of competitions and performance-based activities.

\section{The importance of a quality physical education (QPE)}

In order to tackle public health alarms about the evident low levels of PA among young people broad policy including QPE is essential. QPE is defined as a planned, progressive, and inclusive learning experience in early childhood, primary and secondary education. For many young people, particularly those from less privileged backgrounds, PE represents their only routine of PA (Association for Physical Education, 2008).

Thus, QPE should help establishing a lifelong engagement in PA. The learning experiences proposed to children and adolescents through PE lessons must be developmentally suitable to help them obtain the psychomotricity, the cognitive understanding, and the social and emotional abilities necessary for a physically active life 
(Centers for Disease Control and Prevention, 2010; Harris, 2015; Van Dusen, Kelder, Kohl, Ranjit, \& Perry, 2011).

Besides to receiving valuable learning opportunities in QPE, many other occasions to be physically active within the school setting should be offered. A very effective method of increasing PA opportunities in schools should be installed through a whole school approach to activity and health promotion. Such a concept has a wider focus and involves the creation of a school philosophy and environment which supports and facilitates PA for all pupils and teaching staff (Harris, 2015).

In order to use QPE to the attainment of healthy lifelong habits, PE curricula should be flexible, and accessible to modification, so PE staff are allowed to adapt it to the different needs of their pupils. Meetings with the students could help to guarantee their interests and in order to reflect their needs, and to reinforce wider community commitment through PA.

As the main purposes of PE do not relate only to the promotion of PA and because the time available for PE classes is limited, actually the best PE program does not offer enough PA in order to meet the recommendations for healthy and active lifestyle. The quality of QPE lessons may be measured by the level of PA, but children and adolescents also need to develop movement and behavioural abilities that empower them to be active in and outside the school context. Consequently, alongside QPE, supplementary extra-curricular approaches for promoting PA, complementary to PE should be encouraged: dynamic lunch breaks and playgrounds, active traveling to school, after-school sports and PA, and finally, a health education policy.

Primary school symbolises a perfect period for children to develop competency in locomotor and object control, basic movement abilities, that can deliver the basis for a physically active life (Lubans, Morgan, Cliff, Barnett, \& Okely, 2010). A current study on the durable effects of school-based interventions in order to increase PA, physical fitness and movement abilities, discovered that the continuation of positive outcomes was strongest for movement skills (Lai et al., 2014), meaning more durability compared to PA where the level is low to moderate until adolescence. Other studies have suggested (Fairclough, Stratton, \& Baldwin, 2002) that a PE concentrated on competitions in team sports may contribute to the diminution of the levels of PA in adolescence. Subsequently, schools should offer a greater choice and practice of lifelong PA (fitness activities related to a healthy lifestyle) that may be undoubtedly taken into adulthood. In addition, PE should increase knowledge on physical fitness, goal setting and self-monitoring of PA.

\section{Contribution of the knowledge on habit for physical activity behaviour}

Understanding how habits are developed may help comprehending that PA can become habitual. Furthermore, that knowledge is useful for designing interventions to rise the levels of participation in PA for health campaigns (promoting PA maintenance for long-term health) and NCT prevention.

Hagger $(2019,2020)$ suggests distinguishing between "behaviour" and "process or psychological construct" when contemplating habit according to theory and the most recent research. He defines habit "as a specific action or behavioural tendency that is enacted with little conscious awareness or reflection, in response to a specific set of associated conditions or contextual cues" (Hagger, 2019, p. 119). Additionally, Hagger 
(2020) recommends including in the definition of habit that "any behaviour can be said to be habitually executed where habit plays some facilitating role. This allows for performances that are partly driven by habit, and partly consciously regulated" (Gardner, 2019, cited in Hagger, 2020, p. 3). Moreover, "qualifying definitions of habits with information on the relative control over actions by automatic and conscious processes, and instigation and execution, may unify the definitions of habit with contemporary theoretical views" (Hagger, 2020, p. 3).

The promotion of a healthy and active lifestyle habit in young people, requires an improvement of the measures of habit and methods to more efficiently analyse habits, particularly habit progress and maintenance.

It is improbable that complex and multifaceted behaviours like PA are completely directed by non-conscious processes. PA is often considered at the macro level by research, but rarely the subactions that comprise those actions are being considered. PA is a prominent illustration of multiple behaviours as it includes a series of coordinated actions that establish the behaviour (Hagger, 2019, 2020).

It is important to denote that behaviours like PA need to coordinate the sets of subactions to achieve, but also to organise, to anticipate, and to make the correct decisions in order to get started. When developing a habit for PA, the enormous effort and deliberations engaged in the decision to practice PA are probably becoming less so. Once having undertaken all the deliberations for taking the decision to go swimming (for example: balancing pros and cons; finding the time, place, clothes, etc.), we tend to become less dependent on such thought processes and start to decide quicker and more efficiently based on 'saved' images of the behaviour (Hagger, 2020).

PA habits are established through recurring PA practice in stable contexts (Gardner \& Lally, 2018; Hagger 2020; Wood, 2017), but the establishing of PA as a habit is quite variable because of the incidence of several crucial aspects during its development. Lally et al. (2010) reveals that each individual has its own pace in developing habits and that some persons' behaviour never become 'habitual'. Additionally, the consistency in performing PA is an important factor in the habit development. Hence, the importance of expanding the range and frequency of PA practice in schools. In this sense, Armitage (2005) and Kaushal and Rhodes (2015) reveal that PA habits can develop after some weeks, taking into account of course the individual differences. Nevertheless, only few longitudinal studies have examined habit development over time and consequently more research is needed.

\section{Breaking physical inactivity behaviour}

As beforementioned, and since PA habits foreseeably develop through recurrent participation concomitant with cues or contextual features, the promotion of PA through PE classes should enhance regular and effective practice at school and in extra-curricular situations. Supposing that new behaviours are probable to be controlled by volitional processes from the beginning, such PE programs should firstly concentrate on increasing the motivation to practice PA among children and adolescents. Secondly, a classification of well-defined standards that represent success is required. Positive feedback and goal setting should be used as strategies for that purpose. When children and adolescents have frequent and positive PA experiences during PE classes, they tend to develop these habits. QPE efforts to promote PA habits should consequently motivate 
and encourage students to define and build up PA routines. Moreover, they should be guided in order to achieve them on a regular basis and to check the routine for regularity. As the PA habit advances, the repeated behaviour changes its control from a more reasoned process to a more automatic procedure (Hagger, 2019; Harkin et al., 2016).

In order to break the habit of low PA practice among children and adolescents, PE teachers must help their students to struggle consciously and strongly against their typical effortless response, which is well-learned and already automatic. A QPE needs to make children and adolescents aware and motivated for doing things differently from the beginning. PE teachers must communicate adequately with their students in order help them setting up clear intentions or goals for engaging in a regular PA in the future. Young people need to be guided on how to engage in PA and be provided with positive experiences (Johnson, Wolf, \& Maio, 2017; Naab \& Schnauber, 2016; Prestwich et al., 2014).

It is important to denote that establishing PA habits should include both encouragement by PE teachers through a QPE and a regular practice of PA. As mentioned, $\mathrm{PE}$ teachers are the door keepers of their students when it comes to motivate them "to counteract unwanted habits, particularly when cues and prompts to the undesired behaviour are omnipresent in our environment" (Hagger, 2019, p. 123). Promoting successfully PA habits in children and adolescents and the settings in the environment and the characteristics of the individual that may obstruct or simplify habit development, need to be taken into account by PE teachers. They must be key drivers of physical and health literacy and behaviour change to optimize the PA of children and adolescents. At the same time, parents need to be informed about the benefits of a regular PA practice and encouraged to get involved in school-based PA and events.

\section{END NOTES}

Research on habits has helped to understand how PA habits are initiated, but future research needs to bring more knowledge on how healthy habits are maintained over time and new theories need to integrate multiple perspectives. The QPE needs to be considered as an effective PA promotion among children and adolescents within and outside school contexts.

\section{REFERENCES}

Armitage, C. J. (2005). Can the theory of planned behavior predict the maintenance of physical activity? Health Psychology, 24(3), 235-245.

Association for Physical Education (afPE) (2008). Health Position Paper. Worcester, af PE.

European Commission (2008). EU Physical Activity Guidelines. Recommended Policy Actions in Support of Health-Enhancing Physical Activity. Brussels.

Ferriz, R., González-Cutre, D., Sicilia, Á., \& Hagger, M. S. (2016). Predicting healthy and unhealthy behaviors through physical education: A self-determination theory-based longitudinal approach. Scandinavian Journal of Medicine \& Science in Sports, 26(5), 579-592.

Harris, J. (2015). Association for Physical Education Health Position Paper 2015. Loughborough University Institutional Repository. Association for Physical Education (AfPE).

Galan-López, P., Sánchez-Oliver, A. J., Ries, F., \& González-Jurado, J. A. (2019). Mediterranean Diet, Physical Fitness and Body Composition in Sevillian Adolescents: A Healthy Lifestyle. Nutrients, 11(9). 
Galan-Lopez, P., Sánchez-Oliver, A. J., Pihu, M., Gisladottir, T., Dominguez, R., \& Ries, F. (2020). Association between Adherence to the Mediterranean Diet and Physical Fitness with Body Composition Parameters in 1717 European Adolescents: The AdolesHealth Study. Nutrients, 12(1), 77.

Gardner, B., \& Lally, P. (2018). Modelling habit formation and its determinants. In: Verplanken, B. (Ed.), The psychology of habit. Cham, Switzerland: Springer, 207-229.

Gardner, B., Rebar, A., \& Lally, P. (2019). 'Habitually deciding' or 'habitually doing'? A response to Hagger. Psychology of Sport \& Exercise.

Hagger, M. S. (2019). Habit and physical activity: Theoretical advances, practical implications, and agenda for future research. Psychology of Sport and Exercise, 42, 118-129.

Hagger, M. S. (2020). Redefining habits and linking habits with other implicit processes. Psychology of Sport \& Exercise, 46, 101-106.

Harkin, B., Webb, T. L., Chang, B. P. I., Prestwich, A., Conner, M., Kellar, I., ... Sheeran, P. (2016). Does monitoring goal progress promote goal attainment? A meta-analysis of the experimental evidence. Psychological Bulletin, 142(2), 198-229.

Kaushal, N., \& Rhodes, R. E. (2015). Exercise habit formation in new gym members: A longitudinal study. Journal of Behavioral Medicine, 38(4), 652-663.

Johnson, B. T., Wolf, L., \& Maio, G. (2017). Persuasive communication influences on attitudes. In: Johnson, B. T. \& Albarracín, D. (Eds.), The handbook of attitudes. New York, NY: Psychology Press.

Lai, S. K., Costigan, S. A., Morgan, P. J., et al. (2014). Do school-based interventions focusing on physical activity, fitness, or fundamental movement skill competency produce a sustained impact in these outcomes in children and adolescents? A systematic review of follow-up studies. Sports Medicine, 44, 67-79.

Lally, P., van Jaarsveld, C. H. M., Potts, H. W. W., \& Wardle, J. (2010). How are habits formed: Modelling habit formation in the real world. European Journal of Social Psychology, 40(6), 998-1009.

Lubans, D. R., Morgan, P. J., Cliff, D. P., Barnett, L. M., \& Okely, A. D. (2010). Fundamental movement skills in children and adolescents: Review of associated health benefits. Sports Medicine, 40(12), 1019-1035.

Naab, T. K., \& Schnauber, A. (2016). Validating and refining the response-frequency measure of media habit. Unpublished manuscript, Department of Media, Knowledge and Communication, University of Augsburg, Augsburg, Germany.

Ng, M., Fleming, T., Robinson, M., Thomson, B., Graetz, N., Margono, C., Mullany, E. C., Biryukov, S., Abbafati, C., Abera, S. F., et al. (2014). Global, regional, and national prevalence of overweight and obesity in children and adults during 1980-2013: A systematic analysis for the Global Burden of Disease Study 2013. Lancet, 384, 766-781.

Prestwich, A., Kellar, I., Parker, R., Mac. Rae, S., Learmonth, M., Sykes, B., ... Castle, H. (2014). How can self-efficacy be increased? Meta-analysis of dietary interventions. Health Psychology Review, 8(3), 270-285.

Solmon, M. A. (2015). Optimizing the Role of Physical Education in Promoting Physical Activity: A Social-Ecological Approach. Research Quarterly for Exercise and Sport, 86(4), 329-337.

Van Dusen, D. P., Kelder, S. H., Kohl, H. W., Ranjit, N., \& Perry, C. L. (2011). Associations of physical fitness and academic performance among schoolchildren. The Journal of School Health, 81(12), 733-740.

WHO (2010). Global Recommendations on Physical Activity for Health. Available online: https: / / www.who.int/dietphysicalactivity/global-PA-recs-2010.pdf (accessed on 9 March 2020).

WHO (2019). Report of the Commission on Ending Childhood Obesity: Implementation Plan. Available online: https://www.who.int/end-childhood-obesity/publications/echo -plan-executive-summary/en (accessed on 8 March 2020).

Wood, W. (2017). Habit in personality and social psychology. Personality and Social Psychology Review, 21(4), 389-403. 